\title{
Nutrição e tensão pré-menstrual: preferências alimentares e aspectos fisiológicos
}

\author{
Nutrition and premenstrual tension: food preferences and physiological aspects involved \\ Nutrición y tensión premenstrual: preferencias alimentarias y aspectos fisiológicos involucrados
}

Francisca Karoline Da Costa Silva

ORCID: https://orcid.org/0000-0001-5477-4130

Centro Universitário Estácio do Ceará, Brasil E-mail: karolinesilva684@gmail.com

Moisés Iasley Lima Vasconcelos

ORCID: https://orcid.org/0000-0002-8724-1063 Centro Universitário Estácio do Ceará, Brasil E-mail: moisesasley@gmail.com

Igor de Codes Soares

ORCID: https://orcid.org/0000-0002-8311-629X Universidade de Fortaleza, Brasil E-mail: igordecodes97@gmail.com

Lorena Lopes Brito

ORCID: https://orcid.org/0000-0001-6313-1794 Universidade Estadual do Ceará, Brasil E-mail: lores.brito@aluno.uece.br

João Emanuel Dias Tavares

ORCID: https://orcid.org/0000-0002-4357-0383 Universidade de Fortaleza, Brasil E-mail: joaoemanoel1712@gmail.com Natan Ivens Castro de Sousa

ORCID: https://orcid.org/0000-0001-9863-4594 Universidade Estadual do Ceará, Brasil E-mail: Natan.ivens@aluno.uece.br

Luthyane Andrade Nascimento ORCID:https://orcid.org/0000-0002-7473-7940 Centro Universitário Estácio do Ceará, Brasil E-mail:luthyane.andrade@gmail.com

Francisca Bianca Silva Araújo

ORCID: https://orcid.org/0000-0003-4250-0559 Centro Universitário Estácio do Ceará, Brasil E-mail:fbiancaaraujo@gmail.com Yasmin Dias Pinto Bezerra

ORCID: https://orcid.org/0000-0002-4449-321X Centro Universitário Estácio do Ceará, Brasil E-mail:yasmiindias2002@gmail.com

\section{Resumo}

O estudo tem como objetivo utilizar a literatura como referência para discorrer acerca de aspectos importantes sobre a temática da Tensão pré-menstrual, de forma abrangente e organizada, com enfoque nutricional. Trata-se de um uma revisão integrativa, em que a busca foi realizada nas bases de dados: Pubmed, LILACS e SciELO, utilizando os seguintes descritores disponíveis nos Descritores em Ciências da Saúde (DeCS): Síndrome pré-menstrual, Terapia nutricional e Comportamento alimentar. Foram utilizados os operadores booleanos "AND" e "OR", realizando diferentes combinações. Durante o período reprodutivo da mulher, muitos dos problemas que afetam sua saúde estão associados com as oscilações hormonais relacionadas ao período menstrual. Durante essa fase algumas mulheres podem apresentar diversos sintomas, como: depressão, ansiedade, cólicas, irritabilidade, fadiga, mudança comportamental, cefaléia, dores musculares e retenção hídrica. Nesse sentido, é necessário verificar a influência alimentar na saúde da mulher durante esse período e como essas mudanças afetam sua rotina. A alteração do comportamento alimentar é comum nos dias de TPM, pois há intensificação do interesse por alimentos ultraprocessados, que são ricos em açúcares e gorduras, o que pode ocasionar consequências negativas para a saúde da mulher. Assim, é preciso que as mulheres busquem manter uma boa alimentação e o controle do estresse. Uma dieta composta por hortaliças, fontes de proteína magra, grãos integrais e alimentos com baixo teor de lipídeos, pode contribuir para alívio nos sintomas. Portanto, é lícito que uma alimentação equilibrada em macro e micronutrientes afeta de forma positiva a saúde da mulher no seu ciclo menstrual.

Palavras-chave: Síndrome pré-menstrual; Terapia nutricional; Ciclo menstrual. 


\begin{abstract}
The study aims to use the literature as a reference to discuss important aspects on the topic of premenstrual tension, in a comprehensive and organized way, with a nutritional focus. This is an integrative review, in which the search was performed in the following databases: Pubmed, LILACS and SciELO, using the following descriptors available in the Health Sciences Descriptors (DeCS): Premenstrual syndrome, Nutritional therapy and behavior to feed. The Boolean operators "AND" and "OR" were used, performing different combinations. During a woman's reproductive period, many of the problems that affect her health are associated with hormonal fluctuations related to the menstrual period. During this phase, some women may have different symptoms, such as: depression, anxiety, cramps, irritability, fatigue, behavioral change, headache, muscle pain and water retention. In this sense, it is necessary to verify the influence of food on women's health during this period and how these changes affect their routine. Changes in eating behavior are common on PMS days, as interest in ultra-processed foods, which are rich in sugars and fats, is intensified, which can have negative consequences for women's health. Thus, it is necessary that women seek to maintain a good diet and control stress. A diet consisting of vegetables, lean protein sources, whole grains, and lowfat foods can all contribute to symptom relief. Therefore, it is legitimate that a balanced diet in macro and micronutrients positively affects the health of women during their menstrual cycle.
\end{abstract}

Keywords: Premenstrual syndrome; Nutritional therapy; Menstrual Cycle.

\title{
Resumen
}

El estudio tiene como objetivo utilizar la literatura como referencia para discutir aspectos importantes sobre el tema de la tensión premenstrual, de manera integral y organizada, con un enfoque nutricional. Se trata de una revisión integradora, en la que la búsqueda se realizó en las siguientes bases de datos: Pubmed, LILACS y SciELO, utilizando los siguientes descriptores disponibles en los Descriptores de Ciencias de la Salud (DeCS): Síndrome premenstrual, Terapia nutricional y conducta para alimentar. Se utilizaron los operadores booleanos "Y" y "O", realizando diferentes combinaciones. Durante el período reproductivo de una mujer, muchos de los problemas que afectan su salud están asociados con fluctuaciones hormonales relacionadas con el período menstrual. Durante esta fase, algunas mujeres pueden presentar diferentes síntomas, como: depresión, ansiedad, calambres, irritabilidad, fatiga, cambio de comportamiento, dolor de cabeza, dolores musculares y retención de líquidos. En este sentido, es necesario verificar la influencia de la alimentación en la salud de las mujeres durante este período y cómo estos cambios afectan su rutina. Los cambios en la conducta alimentaria son habituales en los días del síndrome premenstrual, ya que se intensifica el interés por los alimentos ultraprocesados, ricos en azúcares y grasas, lo que puede tener consecuencias negativas para la salud de la mujer. Por ello, es necesario que las mujeres busquen mantener una buena alimentación y controlar el estrés. Una dieta que consista en verduras, fuentes de proteínas magras, cereales integrales y alimentos bajos en grasas puede contribuir al alivio de los síntomas. Por tanto, es legítimo que una dieta equilibrada en macro y micronutrientes afecte positivamente la salud de la mujer durante su ciclo menstrual.

Palabras clave: Síndrome pré-menstrual; Terapia nutricional; Ciclo menstrual.

\section{Introdução}

A menstruação é um fenômeno que dura aproximadamente cinco dias, sendo composto por muco, líquido tecidual e células epiteliais do endométrio que foi descamado. Essa descamação ocorre devido a níveis baixos dos hormônios progesterona e estrogênio. Já o ciclo menstrual tem duração média de 28 dias, podendo haver uma variação entre 20 até 35 dias, em algumas mulheres essa variação pode chegar até 45 dias (Guyton \& Hall, 2011; Tortora \& Derrickson, 2016; Silverthon, 2017).

A Tensão Pré-Menstrual (TPM) ou também conhecida Síndrome Pré-Menstrual (SPM) é caracterizada por sintomas físicos, comportamentais e emocionais que sucedem de modo rotineiro. Os seus sintomas podem ter início em torno de 1 até 2 semanas antes do início da menstruação e reduzem após a chegada do fluxo menstrual, sendo a menstruação um fenômeno caracterizado pelo sangramento e que dura aproximadamente 7 dias, mas pode variar de organismo para organismo (Marván \& Cortés-Iniestra, 2001; Projeto Diretriz, 2011, Silverthorn, 2017).

As manifestações características da TPM podem apresentar-se em aproximadamente $80 \%$ das mulheres, expressando uma imensa variedade de sintomas descritos, além de variações na sua extensão e gravidade com o que ocorre. Desta maneira, os sintomas podem ser diferenciados de acordo com a intensidade com que ocorrem, sendo os sintomas leves aqueles pouco notados pelas mulheres; os sintomas moderados são aqueles que podem causar algum tipo de incomodo, mas sem prejudicar as atividades de rotina e os sintomas de intensidade grave são os que provocam prejuízo nas atividades rotineiras da mulher. Em 
torno de 10\% das mulheres acometidas por TPM afirmam que os sintomas são perturbadores (Valadares et al., 2006; Maia et al., 2014; Projeto Diretriz, 2011).

Diferentes fatores, dentre eles os ambientais, endócrinos e psicológicos, podem interferir de maneira negativa nas relações e na convivência da mulher acometida por TPM, levando inclusive a interferência na vida profissional. Os seus sintomas são inúmeros, mas podemos mencionar, por exemplo, a presença de edema, distensão abdominal, cefaleia, irritabilidade, diarreia e até mesmo a constipação intestinal, entre outros sintomas. As oscilações hormonais em interação com o Sistema Nervoso Central (SNC) são capazes de desestabilizar a mulher acometida por TPM, podendo levar inclusive a quadros de ansiedade, depressão, baixa concentração, consequentemente afetando sua qualidade de vida (Maia et Al., 2014; Tortora \& Derrickson, 2016).

Estes sintomas ocorrem no período pertinente à fase lútea e se encerram com o início da fase folicular. Para que possa ser classificada como TPM, a mulher deve possuir sintomas característicos, entretanto não é preciso que eles se repitam. Em alguns casos, pequenas transformações no estilo de vida podem provocar efeitos benéficos (Valadares et al., 2006; Sbem, 2009; Projeto Diretriz, 2011).

Estudos brasileiros relacionando alimentação ao período pré-menstrual ainda são poucos e não há um tratamento específico, visto que existe uma gama de sintomas apesentadas na TPM que variam de mulher para mulher. No entanto algumas condutas estão sendo discutidas e estudadas com o objetivo de minimizar os sintomas apresentados, desta forma os profissionais nutricionistas podem agir recomendando uma alimentação saudável e equilibrada (Projeto Diretriz, 2011; Santos et al., 2011; Geleski et al., 2018).

Desta forma, o estudo tem como finalidade organizar de forma clara e coesa os conteúdos mais relevantes acerca da Nutrição e sua relação com Tensão Pré-Menstrual, bem como a alimentação e aspectos fisiológicos envolvidos.

\section{Metodologia}

O presente estudo trata-se de uma revisão integrativa, segundo Ercole, Melo \& Alcoforado (2014) esse é um método que busca unificar os diversos resultados encontrados em pesquisas sobre um determinado tema, de maneira sistemática, alinhada e abrangente. Este tipo de estudo recebe esse nome, pois oferece informações amplas sobre um assunto, gerando um corpo de conhecimento. Desta forma buscou-se organizar e agrupar os resultados acerca da Tensão Pré-Menstrual, de maneira abrangente, ordenada e alinhada.

O levantamento bibliográfico ocorreu de Fevereiro de 2021 a Setembro de 2021. A busca pelos artigos selecionados ocorreu por meio das bases de dados científicos: PubMed, LILACS e SciELO, foram utilizados os seguintes descritores disponíveis nos Descritores em Ciências da Saúde (DeCS): Síndrome pré-menstrual, Terapia nutricional e Comportamento alimentar. Foram utilizados os operadores booleanos "AND" e "OR", realizando combinações nas bases de dados citadas anteriormente.

Essas bases de dados foram escolhidas por serem abrangentes, relevantes e por possuir um maior alcance de resultados na área da saúde e nutrição. Foram considerados como critério de inclusão os estudos mais relevantes acerca da Tensão Pré-Menstrual, dos últimos 10 anos, nos idiomas português, inglês e espanhol, e que fossem do tipo transversal, descritivo, qualitativo e quantitativo. Os critérios de exclusão foram: relatos de casos, relatos de experiências, estudos randomizados e de abordagem ecológica.

Posteriormente, foi realizada a leitura dos títulos e resumos para que fossem selecionados os estudos que mais contribuíssem para a elaboração desse trabalho, em seguida ocorreu à leitura completa dos materiais selecionados previamente para a elaboração desse estudo. Após o emprego dos critérios utilizados, foram selecionados 10 artigos. Abaixo podemos 
visualizar o fluxograma acerca dos artigos:

Figura 1. Fluxograma de seleção dos artigos.

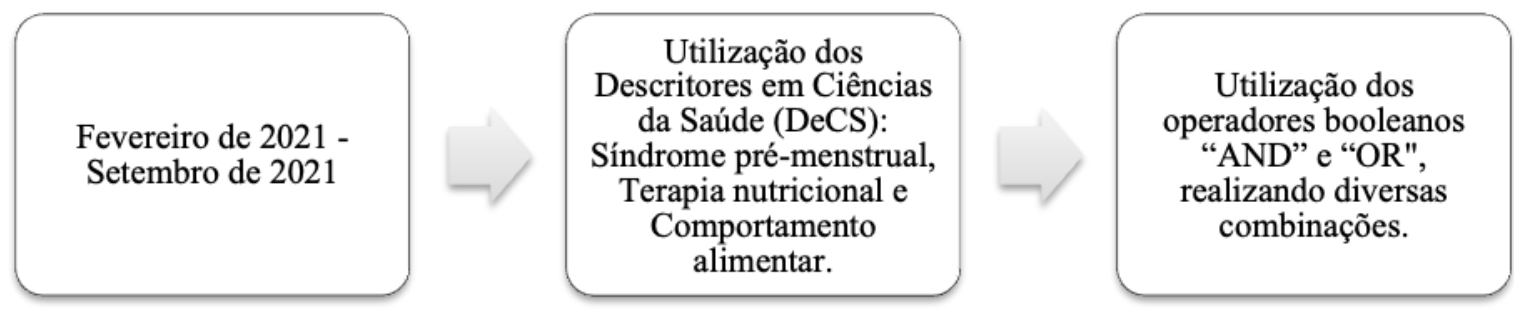

Plataformas Utilizadas: PubMed, LILACS e SciELO

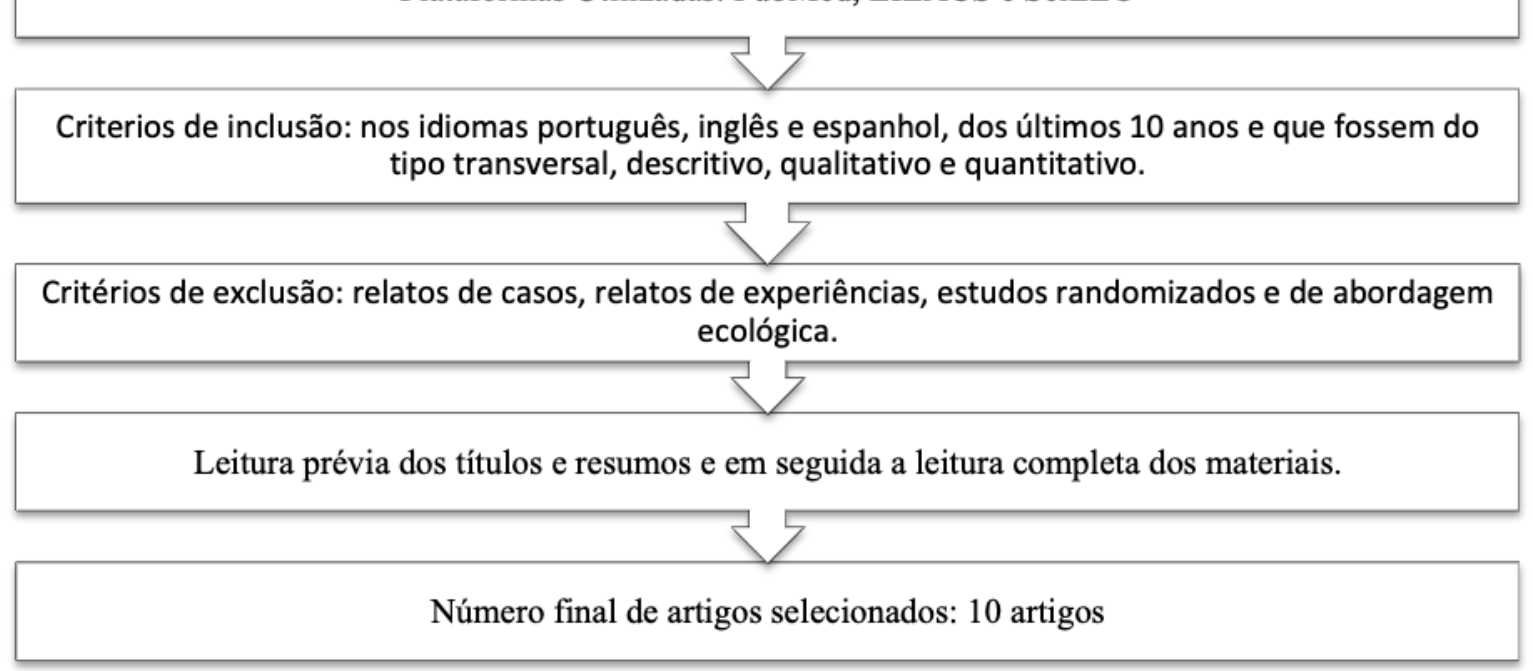

Fonte: Autores.

\section{Resultados e Discussão}

Foi selecionado um total de 10 artigos dos últimos 10 anos. O maior quantitativo de artigos selecionados foi do ano de 2019. Para complementar ainda mais o estudo, foi utilizada uma Diretriz da Federação Brasileira de Associações de Ginecologia e Obstetrícia - FEBRASGO e um Manual de Ginecologia da Associação de Ginecologia e Obstetrícia do Distrito Federal - SGOB. 
Quadro 1- Artigos incluídos na revisão integrativa em relação ao ano de publicação, autores, título, objetivo e principais resultados encontrados:

\begin{tabular}{|c|c|c|c|c|}
\hline Ano & Autor & Título & Objetivo & Principais resultados \\
\hline 2011 & $\begin{array}{c}\text { Lete et } \\
\text { al }\end{array}$ & $\begin{array}{lr}\text { Attitudes of } & \begin{array}{r}\text { Spanish } \\
\text { toward }\end{array} \\
\text { women } & \\
\text { premenstrual symptoms, } \\
\text { premenstrual } \\
\text { and syndrome } \\
\text { dysphoric premenstrual } \\
\text { results of a nationwide } \\
\text { survey }\end{array}$ & $\begin{array}{l}\text { Avaliar as atitudes de } \\
\text { mulheres com sintomas } \\
\text { pré-menstruais em } \\
\text { relação à percepção de } \\
\text { queixas e solicitação de } \\
\text { orientação médica. }\end{array}$ & $\begin{array}{l}\text { A prevalência de síndrome pré-menstrual } \\
\text { moderada ou grave (SPM) foi de } 8,9 \% \text {. Apenas } \\
291(18,7 \%) \text { mulheres buscaram orientação } \\
\text { médica. O principal motivo alegado por } 90,6 \% \\
\text { das mulheres sintomáticas para não procurarem } \\
\text { consulta médica foi considerar que os sintomas } \\
\text { eram normais. }\end{array}$ \\
\hline 2013 & $\begin{array}{c}\text { De } \\
\text { oliveira } \\
\text { et al }\end{array}$ & $\begin{array}{l}\text { Síndrome pré-menstrual } \\
\text { e aspectos relacionados à } \\
\text { antropometria e ao } \\
\text { comportamento } \\
\text { alimentar. }\end{array}$ & $\begin{array}{l}\text { O propósito deste estudo } \\
\text { foi caracterizar o ciclo } \\
\text { menstrual no tocante à } \\
\text { SPM, à antropometria e } \\
\text { ao consumo alimentar. }\end{array}$ & $\begin{array}{l}\text { Verificou-se maior média de circunferência de } \\
\text { cintura e de percentual de gordura corporal na } \\
\text { fase lútea, maior frequência de consumo } \\
\text { excessivo de doces, chocolate, refrigerantes do } \\
\text { tipo diet e café nessa fase, apesar da similaridade } \\
\text { na ingestão de macro e micronutrientes. }\end{array}$ \\
\hline
\end{tabular}

2014 Tolossa Prevalence, impacts and e medical managements of Bekele premenstrual syndrome among female students: cross-sectional study in college of health sciences, Mekelle University, Mekelle, Northern Ethiopia
Avaliar a prevalência, os impactos e os manejos médicos da TPM em estudantes de medicina do Mekelle University College of Health Sciences.
Entre as participantes, $83,2 \%$ tiveram pelo menos um sintoma de PM durante o período menstrual. A prevalência de SPM de acordo com o DSM-IV foi de $37,0 \%$. Cerca de $28,3 \%$ relataram faltas frequentes às aulas, 9,8\% faltas ao exame, $8,1 \%$ notas baixas e $1,7 \%$ deles relataram abandono do aprendizado associado à TPM. Apenas 48,0\% participantes procuraram tratamento médico para a TPM. As modalidades de tratamento utilizadas foram analgésicos, $36,4 \%$, bebidas quentes como café e chá, 7,5\%, e massoterapia e exercícios físicos, $4,0 \%$.

Os resultados relataram que as mulheres apresentam STPM com intensidade diferente, os sintomas variam entre físicos e mentais e interferem no desempenho diário das mulheres.

\section{Souza et al \\ Do Food Intake and Food Cravings Change during the Menstrual Cycle of Young Women?}

O objetivo da pesquisa foi identificar e caracterizar as usuárias de um Ambulatório de Saúde do Município de Tupanciretã-RS

Destacando a questão norteadora: Qual o índice da tensão prémenstrual em usuárias do ambulatório municipal de saúde da mulher?

O objetivo do presente estudo foi avaliar as medidas antropométricas, ingestão alimentar e ânsias alimentares durante o ciclo menstrual de estudantes de graduação da faculdade de nutrição.
O desejo por alimentos ricos em açúcar, sal e gordura, como chocolate, salgadinhos, salgadinhos e sobremesas foi maior $(\mathrm{p}<0,05)$ no período pré-menstrual, embora não tenha refletido nem maior ingestão energética nem alteração na distribuição de macronutrientes. Observou-se maior ingestão de carboidratos, proteínas, fibras e cálcio durante a fase lútea. 


\begin{tabular}{cclrl}
\hline 2019 & Alves & Prevalência da tensão & Quantificar a prevalência \\
et al & pré-menstrual \\
& universitárias. & & entre & da TPM entre \\
& & & universitárias.
\end{tabular}

\begin{abstract}
A prevalência da TPM na amostra foi de 87,5\%. $94,1 \%$ das mulheres encontravam-se na faixa etária de 18 a 26 anos. Os sintomas mais prevalentes no estudo foram: estresse, tensão, dores, irritabilidade e mudanças de humor.
\end{abstract}

\begin{tabular}{|c|c|c|}
\hline \multirow[t]{6}{*}{2019} & \multirow{6}{*}{$\begin{array}{c}\text { Hashim } \\
\text { et al }\end{array}$} & Premenstrual Syndrome \\
\hline & & Associated \\
\hline & & Dietary and Lifestyle \\
\hline & & Behaviors \\
\hline & & University Students: A \\
\hline & & $\begin{array}{l}\text { Cross-Sectional Study } \\
\text { from Sharjah, UAE }\end{array}$ \\
\hline
\end{tabular}

Determinar a prevalência e gravidade da SPM entre estudantes universitários em Sharjah, Emirados Árabes Unidos, e esclarecer suas associações com hábitos alimentares, estilo de vida e fatores antropométricos.
No total, $95 \%$ das participantes relataram pelo menos um sintoma de TPM durante o período menstrual. A prevalência de TPM foi de $35,3 \%$, com sintomas leves sendo os mais comumente relatados. A ingestão de alimentos com alto teor calórico / gordura / açúcar / sal foi associada com risco aumentado de relatar sintomas físicos. No entanto, o consumo de frutas foi associado a uma diminuição do risco de relatar sintomas comportamentais.

\section{Ferraz \\ Avaliação dos aspectos
nutricionais preferências alimentares
relacionadas à tensão
pré-menstrual preferências alimentares
relacionadas à tensão
pré-menstrual preferências alimentares
relacionadas à tensão
pré-menstrual \\ 2019}

Relacionar os aspectos nutricionais preferências alimentares com a tensão prémenstrual de mulheres em um consultório nutricional na cidade de São Bernardo do Campo-SP

\begin{tabular}{|c|c|c|c|}
\hline \multirow[t]{2}{*}{2020} & $\begin{array}{l}\text { Dos } \\
\text { Santos } \\
\text { et al. }\end{array}$ & $\begin{array}{lr}\text { Tratamento alternativo } \\
\text { da síndrome de tensão } \\
\text { pré-menstrual }\end{array}$ & $\begin{array}{lr}\text { O objetivo do estudo foi } \\
\text { investigar } & \text { pacientes } \\
\text { diagnosticadas } & \text { com }\end{array}$ \\
\hline & & $\begin{array}{l}\text { camomila, maçã e } \\
\text { maracujá. }\end{array}$ & $\begin{array}{l}\text { STPM com o uso } \\
\text { associado da camomila } \\
\text { maçã e maracujá. }\end{array}$ \\
\hline 2021 & $\begin{array}{c}\text { Lima, } \\
\text { Facudo } \\
\text { Romão } \\
\& \\
\text { Gouvei } \\
\text { a }\end{array}$ & $\begin{array}{l}\text { Prevalência da Síndrome } \\
\text { Pré-Menstrual } \\
\text { mulheres em idade } \\
\text { reprodutiva }\end{array}$ & $\begin{array}{l}\text { Verificar a prevalência } \\
\text { da SPM em mulheres em } \\
\text { idade reprodutiva da } \\
\text { cidade de Parnaíba-PI } \\
\text { bem como traçar o perfil } \\
\text { clínico e } \\
\text { sociodemográfico das } \\
\text { mulheres com síndrome } \\
\text { pré-menstrual }\end{array}$ \\
\hline
\end{tabular}

A maioria das mulheres afirmou apresentar mudanças no comportamento alimentar durante a TPM. Quanto ao consumo de doces, também a maioria das mulheres disse ter mais vontade de consumir esses alimentos, em especial o chocolate. Grande parte da amostra relatou alteração de humor durante a TPM, além do aumento de peso.
Os parâmetros: irritabilidade e nervosismo, ansiedade e desejo aumentado de comer doce, foram os mais afetados em relação à terapia alternativa. Além disso, foi verificado que $93 \%$ das pacientes citaram ter mais de 4 em até 10 parâmetros afetados.

O estudo apontou uma prevalência de 99,6\% para mulheres com a SPM, sendo maior parcela desta porcentagem classificada como SPM severa, além de apresentarem distúrbios físicos e emocionais decorrentes da SPM.

Fonte: Autores (2021).

\title{
Ciclo Menstrual
}

O início do período reprodutivo na mulher é marcado pela primeira menstruação, denominada de menarca e ocorre em torno dos 12 anos de idade, sendo a faixa de 8 a 13 anos considerada dentro da normalidade. Este ciclo completo tem duração de aproximadamente 28 dias, no entanto pode haver variação de mulher para mulher, podendo ser curto durando aproximadamente 20 dias ou longo, chegando aos 45 (Guyton \& Hall, 2011; Tortora \& Derrickson, 2016; Silverthorn, 2017).

O ciclo divide-se em quatro fases, são elas: fase menstrual, também denominada de menstruação ocorre aproximadamente durante os cinco primeiros dias do ciclo menstrual. A fase pré-ovulatória é a fase que possui uma maior variação de duração. Os níveis elevados de estrogênio no final da fase pré-ovulatória desencadeia a ovulação devido a indução de um feedback positivo nas células que liberam o hormônio luteinizante (LH) e hormônio liberador de gonadotropina, 
(GnRH). A fase de ovulação ocorre cerca de 24 horas após o pico de LH, que ocorre devido aos níveis elevados de estrogênio no sangue. A fase pós-ovulatória é a última fase do ciclo e ocorre antes da fase menstrual, sendo a mais constante (Moore \& Persaud, 2013; Tortora \& Derrickson, 2016).

Em relação aos acontecimentos nos folículos, denominado de ciclo ovariano dividindo-se em três fases, sendo elas: A fase folicular, que é a primeira fase do ciclo, onde ocorre o crescimento folicular no ovário, é o período mais variável durando entre 10 e 21 dias. Na fase da ovulação é onde há o amadurecimento de um ou mais folículos ovarianos e o ovário libera o ovócito. A fase lútea corresponde à fase pós-ovulatória e é nesta fase que há uma predominância da progesterona. Quando o corpo lúteo se degenera e a produção de hormônios reduz, cerca de dois dias após essa degeneração do corpo lúteo, o endométrio novamente descama e a menstruação volta a iniciar já na fase folicular do próximo ciclo ovulatório (Silverthorn, 2017).

\section{Hormônios Femininos}

Os hormônios são moléculas ou proteínas que ao serem produzidas e liberadas na circulação migram para os tecidos e agem por meio de receptores em células sensíveis a eles, para assim promover alguma alteração nas funções celulares coordenando as atividades metabólicas do tecido em questão. Cada célula possui sua combinação de receptores e hormônios, promovendo a resposta celular para aquele determinado hormônio. Os hormônios possuem como função a regulação de diversas atividades, como a regulação do apetite, fome, comportamento alimentar, desenvolvimento e reprodução, entre outras. Estas atividades podem ser reguladas por apenas um ou mais hormônios (Nelson \& Cox, 2014).

O hormônio liberador de gonadotrofina $(\mathrm{GnRH})$ desencadeia a secreção do hormônio luteinizante (LH) e do hormônio folículo estimulante, conhecido comumente por FSH, que em conjunto promovem a liberação dos hormônios sexuais femininos. O hormônio luteinizante $(\mathrm{LH})$ induz o corpo lúteo a produzir progesterona, enquanto que o hormônio folículo estimulante (FSH) induz a produção de estrogênio através das células foliculares. Sendo o estrogênio e a progesterona os principais hormônios sexuais femininos (Moore \& Persaud, 2013; Tortora \& Derrickson, 2016).

Durante os anos reprodutivos, muitos dos problemas que afetam a saúde das mulheres estão relacionados com as variações hormonais associadas ao período menstrual. O ovário produz estrogênios, especialmente o estradiol e a estrona, e progestogênios, particularmente progesterona que é predominante durante a fase lútea, no entanto, ainda há presença de estrogênio. Alterações nos hormônios estrogênio e progesterona ocasionam o ciclo reprodutivo feminino (Silverthorn, 2017; Mahan \& Raymond, 2018).

\section{Tensão Pré-Menstrual: Prevalência e Causas}

No estudo de Alves et al. (2019) onde buscou-se identificar a prevalência de TPM em universitárias, o percentual encontrado foi de $87,5 \%$, sendo 94,1\% das mulheres com idade entre 18 e 26 anos, constatando assim uma alta predominância neste público. Lima, Facundo Romão \& Gouveia (2021) observaram uma prevalência de 99,6\% no público estudado, sendo categorizada em TPM em leve (9,5\%), TPM moderada (33,0\%) e TPM severa (57,5\%).

Falhas ocorridas na síntese de neurotransmissores, oscilações hormonais e redução nas concentrações de nutrientes como a piridoxina (vitamina B6) e cálcio têm sido relacionadas (Mahan \& Raymond, 2018). Existem diversas teorias que buscam explicar a etiologia da TPM apoiadas por ensaios experimentais e clínicos, no entanto nenhuma teoria consegue explicar a diversidade de sintomas e suas apresentações, não podendo assim apontar uma etiologia (Nogueira \& Pinto e Silva, 2000).

Embora os mecanismos fisiopatológicos não estejam evidentes, sugere-se que modificações ocorridas no funcionamento do sistema nervoso autônomo (SNA) durante a fase lútea tardia podem ter relação com os sintomas 
comportamentais e psicossomáticos presentes no período pré-menstrual do ciclo (Matsumoto et al., 2007).

Segundo Da Silva Guedes; De Alcântara Silva \& De Sales Ferreira (2021) há hipótese de que a TPM pode possuir ligação com o eixo-hipotálamo-hipófise-ovariano levando a uma resposta atípica, devido os índices dos níveis estrógeno e progesterona sobre os neurotransmissores ácido gama-aminobutírico e serotonina.

\section{Sintomatologia Envolvida na Tensão Pré-Menstrual}

A TPM afeta grande parcela do público feminino em idade reprodutiva. Os seus sintomas e alterações são diversos, já foram catalogados cerca de 150 sintomas diferentes que ocorrem da forma mais variada, desta forma, torna-se importante o estudo sobre a alimentação e nutrição (Da Silva Guedes; De Alcântara Silva \& De Sales Ferreira, 2021)

Durante este período algumas mulheres podem desenvolver diversos sintomas, existem muitos sintomas identificados e dentre eles temos o desconforto geral, depressão ansiedade, cólicas, irritabilidade, fadiga, mudança de humor, cefaléia, cãibras, dor na região das mamas, dores musculares e nas articulações e retenção hídrica, compondo assim o grupo de sintomatologia pré-menstrual mais comum entre as mulheres. Durante a fase lútea constata-se uma maior intensidade nos sintomas físicos, além da presença de problemas relacionados ao cotidiano da mulher (Leporace \& Garcia, 2009; Sgob, 2017; De Oliveira et al., 2013; Mahan \& Raymond, 2018).

Tolossa e Bekele (2014) afirmam que existe um impacto negativo no desempenho acadêmico de estudantes quando há presença de sintomas graves, relacionando o período pré-menstrual e sua sintomatologia às faltas, baixo rendimento e abandono, comprometendo o desempenho acadêmico.

A sensação de inchaço pode ser proveniente da retenção de água, causada pelo estrogênio que possui semelhança com a aldosterona. Essas modificações no equilíbrio de água e sódio são conferidas às variações nas concentrações de progesterona e estrogênio durante o ciclo menstrual (Mahan \& Raymond, 2018).

De acordo com a variedade de manifestações presentes no período pré-menstrual, podem-se classificar os sintomas nos tipos A, C, D e H. Os sintomas do tipo A apresentam características de ansiedade, sendo presentes a irritabilidade, alteração do humor e tensão, além de dificuldade para dormir. O tipo C apresenta características de compulsão alimentar, apresentando o desejo por doces, alimentos com alto índice glicêmico e elevado apetite. O tipo D caracteriza-se pela depressão, onde se apresentam os sintomas de baixa autoestima, choro e algumas mulheres passam a se irritar com mais facilidade. $\mathrm{O}$ tipo H destaca a sensação de inchaço, sensibilidade mamária e em outras regiões do corpo, isso devido à tendência de reter líquidos que ocorre neste período (Mattia et al., 2008; Febrasgo, 2017).

Para determinar a presença ou ausência de TPM a Federação Brasileira das Sociedades de Ginecologia e Obstetrícia (FEBRASGO) cita o American College of Obstetricians and Gynecologists (ACOG) onde o critério de base é de que a mulher deve apresentar pelo menos um ou mais sintomas somáticos e/ou emocionais durante o período pré-menstrual ou a fase lútea, estando assintomático na fase folicular e que possa interferir nas atividades de rotina das mulheres (Gaion \& Vieira, 2010; Febrasgo, 2018).

O diagnóstico da TPM é feito pela presença de sintomas leves ou sintomas moderados, em pelo menos cinco dias que antecedem o início da fase menstrual e estando presentes em por pelo menos dois ou três ciclos consecutivos. As mulheres que sofrem de TPM ou também de sua forma mais grave, denominada de Transtorno Disfórico Pré-Menstrual (TDPM), geralmente não procuram um atendimento médico (Lete et al., 2011; Projeto Diretriz, 2011).

\section{Alimentação e Tensão Pré-Menstrual}

Ao longo do período Pré-Menstrual, também pode haver mudanças no estilo e no comportamento alimentar, sendo assim um possível aspecto contribuinte para os desejos alimentares nas mulheres. Em determinadas situações há uma 
intensificação do interesse por alimentos ultraprocessados, por consequência o desejo aumentado por esse tipo de alimento pode vir a ocasionar variações no peso corpóreo, sendo de grande importância a realização periódica de atividade física, sob a orientação de um profissional da área (Souza et al., 2018; Orra \& Ferraz, 2019).

Os resultados encontrados por Souza et al (2018), mostram que as mulheres durante o período pré-menstrual, tendem a ter um desejo aumentado por alimentos ricos em açúcar, sódio e lipídeos, como chocolates, lanches e sobremesas. Os estudos de Hashim et al. (2019) mostraram uma relação negativa entre a gravidade da TPM com os hábitos alimentares, consumo de alimentos de alto valor calórico, gorduras, açúcares e sal e o estilo de vida, por outro lado considerou-se o consumo de frutas como um fator protetor contra a TPM.

Desta maneira, o consumo de frutas pode estar ligado à ingestão de nutrientes como cálcio, magnésio, potássio, zinco, triptofano, piridoxina (B6) entre outros (Cozzolino, 2016). Além disso, o consumo de frutas colabora para a ingestão de fibras alimentares que são benéficas na melhora de alguns sintomas da TPM, como quando há casos de mau funcionamento intestinal (Freitas, 2021).

Dentre os alimentos que contém os nutrientes listados anteriormente, temos a banana, frutas secas, espinafre, brócolis, tomate, semente de abóbora, amêndoas, amendoim, nozes picadas, castanha-do-Brasil, iogurte, leite, queijo, acém cozido, alguns tipos de peixes, bife de fígado, suco de uva, caju e frango (Cozzolino, 2016).

O desejo pelo consumo de alimentos doces em momentos de estresse pode trazer certa sensação de recompensa fisiológica, visto que desta forma há um maior movimento do triptofano, precursor da serotonina, para o cérebro. Desta forma causando a sensação de relaxamento e bem estar. O chocolate é um dos alimentos mais desejados durante o período prémenstrual, este, quando escolhido corretamente pode contribuir para fornecimento vitaminas e minerais, além de melhorar o humor, pois se acredita que componentes do cacau, ingrediente utilizado na sua fabricação, tenha o efeito positivo, aumentando as concentrações de endorfina e serotonina no cérebro (Mahan \& Raymond, 2018).

No estudo de Dos Santos et al. (2020) onde buscou-se alternativas para a melhoria de alguns sintomas, foi observado que a utilização e suco de maracujá e maçã junto com o chá de camomila podem trazer resultados positivos para o tratamento da TPM, como por exemplo, a melhora dos sintomas de irritabilidade, nervosismo, ansiedade e inclusive o choro.

É necessário que as mulheres busquem manter uma boa alimentação, visto que o perfil alimentar da população ocidental é extremamente pobre em frutas, vegetais, grãos e fibras e excessivamente ricos em gordura e sódio. É essencial que haja o controle do estresse no dia a dia a fim de reduzir os impactos no trabalho e estudos. Uma dieta composta por hortaliças, fontes de proteína magra, grãos integrais e alimentos com baixo teor de lipídeos, pode contribuir para alívio nos sintomas relatados por algumas mulheres (Silveira et al., 2014; Mahan \& Raymond, 2018; Vasconcelos et al., 2021).

Segundo Orra \& Ferraz (2019) a literatura cientifica ainda não determina um protocolo que possa ser seguido ou tido como base para o profissional nutricionista, no entanto é importante que o profissional busque formas de realizar durante sua anamnese o rastreamento de sintomas que possam ser utilizados para direcionar sua conduta nutricional, desta maneira, podendo auxiliar na redução de sintomas característicos.

\section{Considerações Finais}

Levando em consideração os aspectos mencionados, está claro que a Tensão-Pré-Menstrual (TPM), é caracterizada por sintomas físicos, comportamentais e emocionais. Um dos sintomas relatados durante a TPM é o comportamento alimentar alterado, pois foi visto uma intensificação do interesse por alimentos ultraprocessados, ricos em açúcares e gorduras, o que pode ocasionar a variações no peso corpóreo. Além disso, observou-se que os diversos sintomas relatados são fatores importantes associados a alterações da dieta, colaborando para o aumento dos hábitos alimentares prejudiciais à saúde. 
Desta maneira, é imprescindível que mulheres busquem manter uma boa alimentação e o controle do estresse, com a finalidade de reduzir os impactos sintomatológicos da TPM no seu dia a dia, dado que uma boa alimentação nutricionalmente balanceada em macro e micronutrientes afeta positivamente a saúde da mulher acometida por sintomas pré-menstruais característicos da TPM, auxiliando na constância saudável do peso e garantindo a manutenção da saúde e qualidade de vida. É necessário que sejam realizados mais estudos relacionando a TPM e a alimentação feminina, com o intuito de realizar mais descobertas acerca desse tema.

\section{Referências}

Alves, M. H. F., Ribeiro, G. O., Vitorino, G. d. S., Andrade, A. A. d., Uchoa, E. P. B. L., \& Carvalho, V. C. P. d. (2019). Prevalência da tensão pré-menstrual entre universitárias. Fisioterapia Brasil, 20(3), 392-399.

Cozzolino, S. M. F. (2016). Biodisponibilidade de nutrientes (5a ed.). Editora Manole.

Silva Guedes, T., De Alcântara Silva, V. L., \& De Sales Ferreira, J. C. (2021). A influência do comportamento alimentar e a utilização dos compostos bioativos na síndrome pré-menstrual. Brazilian Journal of Development, 7(11), 103060-103081.

Oliveira, D. R., Bicalho, A. H., Davis, L. G., Brito, D. A. A., Davis, P. S., \& dos Santos, L. C. (2013). Síndrome pré-menstrual e aspectos relacionados à antropometria e ao comportamento alimentar. O Mundo da Saúde, 37(3), 280-287.

Santos, C. C., Alberton, O., Belettini, S. T., Donadel, G., Dalmagro, M., Otenio, J. K., ... \& Lourenço, E. L. B. (2020). Tratamento alternativo da síndrome de tensão pré-menstrual com camomila, maçã e maracujá. Research, Society and Development, 9(10), e2929108702-e2929108702.

Ercole, F. F., Melo, L. S. D., \& Alcoforado, C. L. G. C. (2014). Revisão integrativa versus revisão sistemática. Revista Mineira de Enfermagem, $18(1)$, 9-12.

Febrasgo. (2017) Tensão Pré-menstrual. https://www.febrasgo.org.br/pt/noticias/item/44-tensao-pre-menstrual.

Febrasgo. (2018). Tensão Pré-Menstrual - Critérios para diagnóstico. https://www.febrasgo.org.br/pt/noticias/item/422-tensao-pre-menstrual-criterios-paradiagnostico.

Freitas, G.B. L. (2021). Pesquisa e Ações em Saúde Pública (2ed) (Cap 8, pp 65- 74). Editora Pasteur.

Gaion, P. A., \& Vieira, L. F. (2010). Prevalência de síndrome pré-menstrual em atletas. Revista Brasileira de Medicina do Esporte, 16, $24-28$.

Geleski, A. C., Benincá, S. C., Vieira, D. G., de Freitas, S. P., \& Mazur, C. E. (2018). Sintomas envolvidos, consumo alimentar e estado nutricional de universitárias durante o período menstrual. Revista de Atenção à Saúde (ISSN 2359-4330), 16(56), 5-11.

Guyton, A. C. \& Hall, J. E (2011). Tratado de fisiologia médica (12a ed). Editora Elsevier.

Hashim, M. S., Obaideen, A. A., Jahrami, H. A., Radwan, H., Hamad, H. J., Owais, A. A., ... \& Al-Yateem, N. (2019). Premenstrual syndrome is associated with dietary and lifestyle behaviors among university students: A cross-sectional study from Sharjah, UAE. Nutrients, 11(8), 1939.

Leporace, C., Garcia, F. (2009) . TPM Sem Tensão. https://www.endocrino.org.br/tpm-sem-tensao/.

Lete, I., Dueñas, J. L., Serrano, I., Doval, J. L., Martínez-Salmeán, J., Coll, C., \& Arbat, A. (2011). Attitudes of Spanish women toward premenstrual symptoms, premenstrual syndrome and premenstrual dysphoric disorder: results of a nationwide survey. European Journal of Obstetrics \& Gynecology and Reproductive Biology, 159(1), 115-118.

Lima, L. M., Facundo Romão, M., \& Gouveia, G. P. de. (2021). Prevalence of Premenstrual Syndrome in women of reproductive age. Research, Society and Development, 10(8), e6510817116.

Mahan, L. K.\& Raymond, J. L (2018). Krause: Alimentos, Nutrição e Dietoterapia (14a ed). Editora Elsevier

Maia, M. d. S., Aguiar, M. I. F. d., Chaves, E. S., \& Rolim, I. L. T. P. (2014). Qualidade de vida de mulheres com tensão pré-menstrual a partir da escala WHOQOL-BREF. Ciência, Cuidado e Saúde, 13(2), 236-244.

Marván, M. L., \& Cortés-Iniestra, S. (2001). Women's beliefs about the prevalence of premenstrual syndrome and biases in recall of premenstrual changes. Health Psychology, 20(4), 276.

Matsumoto, T., Ushiroyama, T., Kimura, T., Hayashi, T., \& Moritani, T. (2007). Altered autonomic nervous system activity as a potential etiological factor of premenstrual syndrome and premenstrual dysphoric disorder. BioPsychoSocial medicine, 1(1), 1-8.

Mattia, A. L. d., Telles, B., Santos, C. A. M., \& Bernauer, M. C. (2008). Síndrome pré-menstrual: influências na equipe de enfermagem de centro cirúrgico. $O$ Mundo da Saúde, 32(4), 495-505.

Moore, K. L. \& Persaud, T. V. N. (2013). Embriologia Básica (8a ed). Editora Elsevier.

Nelson, D. L.\& Cox, M. M (2014). Princípios de Bioquímica de Lehninger. Editora Artmed. 
Nogueira, C. W. M., \& Pinto e Silva, J. L. (2000). Prevalência dos sintomas da síndrome pré-menstrual. Revista brasileira de ginecologia e obstetrícia, 22(6), 347-351.

Orra, L. N., \& Ferraz, R. R. N. (2019). Avaliação dos aspectos nutricionais e preferências alimentares relacionadas à tensão pré-menstrual. International Journal of Health Management Review, 5(2), 1-7.

Projeto Diretriz (2011). Associação Médica Brasileira. Conselho Federal de Medicina. Tensão Pré-menstrual. https://diretrizes.amb.org.br/_BibliotecaAntiga/tensao_pre_menstrual.pdf.

Santos, L. A. S. D., Soares, C., Dias, A. C. G., Penna, N., Castro, A. O. D. S., \& Azeredo, V. B. D. (2011). Estado nutricional e consumo alimentar de mulheres jovens na fase lútea e folicular do ciclo menstrual. Revista de Nutrição, 24(2), 323-331.

Sbem. Síndrome Pré-Menstrual (SPM). Sociedade Brasileira de Endocrinologia e Metabologia, $2009 . \quad$ Disponível em: <https://www.endocrino.org.br/sindrome-pre-menstrual-spm/>. Acesso em: 20 de março de 2021.

Sgob. (Org.) (2017). Manual de Ginecologia da Sociedade de Ginecologia e Obstetrícia de Brasília. Brasília: Luan Comunição. http://www.sgob.org.br/wpcontent/uploads/2017/10/ManualSGOBdigital11102017.pdf.

Silveira, A. D., Vieira, E., Leão, D. M., Nicorena, B. P. B., Rodrigues Ferreira, R., \& Sandoval Longoria, E. (2014). Síndrome de tensión pre-menstrual observada en usuarias del ambulatorio municipal de salud de la mujer. Enfermería Global, 13(35), 63-73.

Silverthorn, D. U (2017). Fisiologia Humana: Uma Abordagem Integrada (7a ed). Editora Artmed.

Souza, L. B. D., Martins, K. A., Cordeiro, M. M., Rodrigues, Y. D. S., Rafacho, B. P. M., \& Bomfim, R. A. (2018). Do food intake and food cravings change during the menstrual cycle of young women?. Revista Brasileira de Ginecologia e Obstetrícia, 40, 686-692.

Tolossa, F. W., \& Bekele, M. L. (2014). Prevalence, impacts and medical managements of premenstrual syndrome among female students: cross-sectional study in college of health sciences, Mekelle University, Mekelle, Northern Ethiopia. BMC women's health, 14(1), 1-9.

Tortora, G. J. \& Derrickson, B (2016). Princípios de anatomia humana (14a ed).Editora Guanabara Koogan.

Valadares, G. C., Ferreira, L. V., Correa Filho, H., \& Romano-Silva, M. A. (2006). Transtorno disfórico pré-menstrual revisão: conceito, história, epidemiologia e etiologia. Archives of Clinical Psychiatry (São Paulo), 33, 117-123.

Vasconcelos, M. I. L. ., Fernandes, H. M. A., Barbosa, E. da S., Grangeiro, R. F. de O. ., Sena, D. B. G. de ., Lopes, V. C. B. ., Sandes, M. de O. ., Sousa, L. S. de, Oliveira, R. G. de, Tabosa, V. L. ., \& Firmino , L. A. R. G. . (2021). Nutrition and chronic kidney disease (CKD): Presentation of new recommendations and food standards by the last scientific evidence. Research, Society and Development, 10(6), e28610615891. 